\title{
Developing a Signal Booster for Improved Communication in Remote Areas
}

\author{
Onyemaobi Bethram Chibuzo (Corresponding author) \\ Department of Computer Science, Salem University, \\ KM 16 Ajaokuta- Lokoja Road, Lokoja Kogi State Nigeria. \\ Tel: +234-803-590-6987 E-mail: conyemaobi@yahoo.com \\ Adebayo Philip Omoniyi \\ School of Engineering, Kogi State Polytehnic, \\ Itakpe Kogi State Nigeria. \\ Tel: +234-803-832-3615 E-mail: philipadebayo41@gmail.com
}

\begin{abstract}
A GSM mobile phone signal booster basically consists of a bidirectional amplifier created to boost weak cell phone signals in remote or hard-to-reach areas. The purpose of boosting signals is to promote clearer reception for cellular phone users in difficult locations. It is meant to solve the problem of bad network in offices, camps, recreational centers, homes and in vehicles. The device brings improved network signal to a relatively poor network area. This work intends to develop a device which can provide users with relatively high signal strength in a poor network area and at a lower cost. This is done in order to provide them with seamless, uninterrupted and reliable communication and in the final analysis, make GSM network available everywhere irrespective of height, terrain and location. To effect this, three major components are utilized, they include: an external antenna to capture the weak signal, a signal amplifier to boost the captured signal and an internal antenna to redistribute the signal for users around the area where enhanced signal is required.
\end{abstract}

Keywords: Amplifier, Antenna, Base Station, GSM, Mobile Phones and Signal Booster

DOI: $10.7176 / \mathrm{NCS} / 10-06$

Publication date:August $31^{\text {st }} 2019$

\section{Introduction}

The GSM mobile phone signal booster is a bidirectional amplifier created to boost weak cell phone signals in remote or hard-to-reach areas. It is a system, which takes mobile phone signal from one area where the signal is active, and repeats (or amplifies) it to an area where the signal is poor. The purpose of boosting signals is to promote clearer reception for cellular phones users in difficult locations [6].

In order for GSM services to be made available everywhere to subscribers with GSM compliant phones, antennas are mounted on masts so that they can transmit and receive radio waves. Radio base stations are sites that enable mobile phones to work. It is composed of several antennas mounted on towers with electronics in it at the base. They can equally be mounted on existing building, rooftops etc. Without base stations, mobile phones will not work. As mobile phone users move away from these base stations, the radio signal also weakens. This is where the need for a signal booster became necessary. The radio link must be made available so that mobile phones can connect to it for the purpose of making calls and harnessing other data services. It is meant to solve the problem of bad network in offices, homes, schools, camps, hospitals, stadiums, rural areas and in vehicles on transit. The device brings improved network capability to relatively poor network areas.

Many things contribute to poor network signals, among them are:

i. The concentration of commercial centers and houses in most rural locations are too low. It is unlikely that the service provider will do anything to improve reception, due to the high cost of erecting a new tower.

ii. Attenuation, a process that leads to weakening of signal strength increases when they come in contact with lead used for roofing material, thick concrete, energy efficient windows and metal window. 
iii. Urban areas, which usually have strong cellular signals throughout, often experience dead zones caused by destructive interference of waves which have taken different paths caused by the signal bouncing off buildings. This is referred to as Multipath interference.

iv. The longer wavelengths have the advantage of being able to diffract to a greater degree, so are less reliant on line of sight to obtain a good signal, but still attenuate significantly [7].

It is no longer news that GSM subscribers have occasional trouble with their services, such as dropped calls or no service which can inevitably lead to loss of useful calls, loss of lives and business opportunities. Rural dwellers, travelers, campers and drivers sometimes find it difficult to make calls due to obstructions posed by mountains, hills, valleys, foliage and other natural obstructions. The result is slower data speeds and connectivity problems. These problems are properly addressed using a mobile phone signal booster. Installing a signal booster will help to maximize cellular phone customer satisfaction. The device consists of an external antenna which serves to capture the low signal from a base station. The amplifier then repeats or boosts the signal and transfers it to an internal antenna. The internal antenna takes the repeated signal and redistributes it so the mobile phones in the vicinity can make calls and explore other data services. In this development, a weak signal (not a stronger signal, is amplified). More often than not, the external antenna is placed in an area particularly in the direction or line of sight with a base station. This work also delved into areas having a significant relevance on the GSM evolving trends and architecture bearing in mind that the entire field of telecommunication is dynamic.

\section{Related Works}

\subsection{The Design and Construction of Wi-Fi Antenna Booster.}

The research work introduced a new design to a device which can provide Wi-Fi users with high signal strength and a lower cost in order to enable them have a seamless, uninterrupted and reliable communication. To implement this, a biquad antenna, a booster circuit and a USB connector were constructed and a Wi-Fi booster system was formed by interconnecting these three circuits together [2].

The project was aimed at designing and constructing a device which will trap and boost received wireless signals. It is known that wireless devices that receive and transmit wireless signals such as wireless cards, routers, access points etc already exists. In fact Wi-Fi antenna boosters also exist. The devices, according to the researcher have their limitations:

i. The wireless signal antennas in a laptop computer are enclosed in the laptop case. This enclosure leads to a bad reception.

ii. The wireless network cards that come inbuilt in laptops, routers, or access points have limited power and range. The solution then lies in getting a wireless card with higher power or attaching an antenna booster to the computer.

The function of the Biquad was to receive signals; these signals were then sent to the booster for amplification. After amplification the signals passed through to the USB connector circuit which is plugged into a computer. Using software to analyze the throughput and signal strength showed a repeated $12 \mathrm{~dB}$ gain. Further test showed that signals which could not be captured by the in-built wireless card of a computer system were captured with this device.

The researcher defined the antenna booster as a device which is designed to amplify reception or received signals so that a marginal signal can be utilized. According to him, the way the antenna booster works is that it expands the area of the antenna, allowing it to pick up more of a weak signal and then translate it into a stronger signal for the device that the antenna is attached to. Antenna boosters can be used to improve radio signals in any frequency band be it the Very Low Frequency (VLF) band or the Extremely High Frequency (ELF) band.

The researcher also opined that the booster finds suitable application in many areas which include but are not limited to the following areas: 
Cell phones: Areas which have poor network coverage require a signal booster (also called an antenna booster or an antenna amplifier). Although it is difficult to attach an antenna booster to a cell phone without making the phone hard to use, an antenna booster works effectively for cell phones (This is the focus of this work).

Television sets: This also requires an antenna booster for us to be able to receive a variety of channels. Depending on the kind of television, the dipole or half-dipole (also known as rabbit ears antenna) which comes with the television is often not used these days by consumers of electronic products.

Boosting of Wi-Fi (Wireless Fidelity) signals: Wi-Fi is a popular technology that allows an electronic device to exchange data wirelessly (using radio waves) over a computer network, including high-speed Internet connections. For instance devices such as a personal computer, video game console, smart phone, tablet, or digital audio player some of which do not have an Ethernet port, most of the times the signals present are not strong enough, in such cases an antenna booster will do [2].

Satellite communication: This uses a form of Low Noise Amplifiers (LNA). Here an LNA having a very low noise figure is required.

He concluded by making it clear that the Wi-Fi booster system was able to trap wireless signals through the

biquad antenna which has a gain of $12 \mathrm{dBi}$ and was amplified by the booster resulting in an overall gain of $24 \mathrm{dBi}$.

\subsection{In-Car Cellular Signal Boosters}

A white paper Prepared for Wilson Electronics and delivered by Andrew M. Seybold, CEO and Principal

Consultant at Robert P. O'Hara Partner, in July 2010 (7) explained the problems that may be encountered while designing an In-Car Cellular Signal Boosters: "Cars with their metal bodies do a great job of blocking radio waves. Thus dropped and missed calls or slow data rates are all too common. The solution to this problem is to boost or amplify the signal between the phone and the cell site. An in-car or mobile cellular signal booster consists of two components: a booster that is placed inside the car (perhaps incorporated into a cradle for the cell phone) and an antenna that is placed outside of the car. The booster amplifies both the signal that the phone receives from the cell site and the signal the phone transmits to the cell site. Thus the phone always has a strong signal (more "bars") and dropped calls are virtually eliminated" [7].

The white paper spelt out problems to overcome in the design of a booster. They include:

i. Oscillation due to feedback must be avoided. If the external antenna is placed too close to the in-car cradle (which has its own internal antenna), oscillation due to feedback can occur, similar to when a microphone is placed too close to the speaker of a public address system and a howling whine comes out of the speakers. In a cell phone booster, feedback oscillation causes the system to generate noise that can interfere with nearby cell sites' ability to receive signals from other cell phones, causing disruption of service to other users.

ii. Overload of the cell site with which the phone is communicating must be prevented. When you are far from the nearest cell site, the booster needs to transmit at the maximum allowable power. If you then drive close to the cell site, it must appropriately adjust itself so as not to overwhelm the cell site and to avoid any type of network overload or potential interference.

iii. Interference to adjacent cell sites must not occur. A more subtle variation of the previous problem occurs when your cell phone is communicating with a distant cell site, but there is another cell site (operated by a different provider) close by. If the booster transmits a strong signal in order to reach the distant site, it could potentially interfere with the nearby cell site. This must not happen.

The solution proffered by Wilson Electronics include:

i. Avoiding oscillation due to feedback. If the external antenna is placed too close to the in-car cradle (which has its own internal antenna), oscillation due to feedback could occur similar to when a microphone is placed too close to the speaker of a public address system and a howling whine comes out of the speakers.

ii. Preventing overload of the cell site with which the phone is communicating. When you are far from the nearest cell site, the booster needs to transmit at the maximum allowable power. If you then drive close to the cell site, it must appropriately adjust itself so as not to overwhelm the cell site. 
iii. Avoiding interference to adjacent cell sites. A more subtle variation of the above occurs when your cell phone is communicating with a distant cell site, but there is another cell site (operated by a different service provider) close by. There is a possibility that the booster could cause interference to the other network operators' cell site. If the booster transmits a strong signal in order to reach the distant site, it could potentially interfere with the nearby cell site [7].

Thus a booster must be designed to avoid transmitting broadband noise that would cause a problem to nearby cell sites.

\subsection{Design and Construction of a Mobile Phone Signal Jammer}

Signal jammer is an electronic device that jams the frequency of a transmitting device. Mobile phone signal

jammer interferes with the communication frequency within a specified radius depending on the strength of the signal jammer. This is achieved by generating and transmitting radio signal of the same frequency as the base station (BS) of the mobile phone and at a higher power so as to collide and cancels out the mobile phone signal. The method employed in realizing this project is made up of discrete components, resistor, capacitor, inductor and transistor to generate the required frequency (noise) and then amplifies the frequency generated to range of $800 \mathrm{MHZ}$ to $1.4 \mathrm{GHZ}$ in order to match the frequency of the mobile phone being transmitted by the base station (BS).

Jamming of a mobile signal is successful, when a mobile phone within the area where the jamming device is situated, is disabled. This was confirmed to be efficient in jamming known GSM providers base station signals and their frequencies, thereby allowing no call to go through.

All of the research works reviewed so far has contributed immensely to the assertion that signals of many

types can be enhanced, boosted or improved upon. This is an enviable trend in the radio frequency and mobile/cellular phone technology. There is so much to benefit and profit from this research area.

\section{Materials and Methods}

\subsection{Component Selection}

The right components were selected based on their design specifications, durability, availability and reliability in order to achieve the construction objectives. Some of the major components are outlined here:

\section{Power Source}

The power source employed in this project work is solar system which is highly preferred by virtue of the fact that the device is meant to be transferable as the need arises.

Solar energy clearly meet the project objectives as it can be used in remote areas especially along the road when on transit, in camps, rural areas etc.

\section{The solar panel}

The solar panel made up of solar cells(also called photovoltaic cells or photoelectric cells) are solid state electrical devices that converts the energy of light directly into electricity by the photovoltaic effect. There are different types of solar cells. They include:

Amorphous Silicon solar cell, Biohybrid solar cell, Concentrated PV cell, crystalline silicon solar cell and Dyesensitized solar cell (DSSC).

The three types of technology utilized in the design of solar panels are: Monocrystalline, Polycrystalline and thin film amorphours. The type employed in this work is polycrystalline solar panel (see figure 1 below). They are made from crystalline silicon. This is the type that is majorly used in residential solar installations. The specification is as listed below:

Power rating is $1.2 \mathrm{~W}$

Voltage is 9V

The current is given by: $\underline{\text { Power rating }(\mathrm{W})}=\underline{1.2}=0.1333 \mathrm{~A}$

$$
\text { Voltage rating (V) } 9
$$




\section{The transistor}

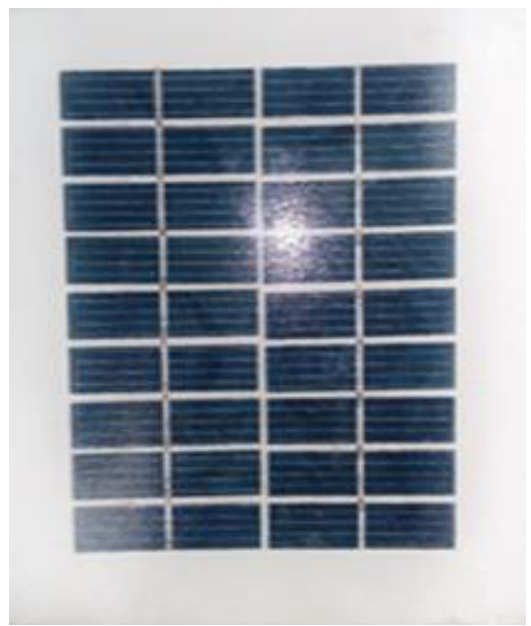

Figure 1. Polycrystalline solar panel

Transistors are switching circuits. A transistor is a semiconductor device used to amplify or switch electronic signals and electrical power. It is composed of semiconductor material usually with three terminals for connection to an external circuit, namely collector, base and emitter [10].

There are two major types of transistors. NPN and PNP transistors. The type used in this project is the NPN transistor - C555IA. The transistor here serves as the core of the signal amplifier. The purpose of an amplifier is to boost the input signal received by the external antenna.

Signal amplifiers provide $20 \mathrm{~dB}$ to $50 \mathrm{~dB}$ gain and many of the more expensive models provide over $50 \mathrm{~dB}$. Excellent high-power models (used by commercial operators) offer a gain of around 100dB. Since decibels are logarithmic, a $30 \mathrm{~dB}$ gain represents a one thousand fold signal power increase - meaning the total amplification of a booster with greater than around $50 \mathrm{~dB}$ is likely to be useless without a good, well aligned antenna. This is due to the difficulty of filtering the correct signal out from the background noise.

\section{Integrated circuit}

An integrated circuit, or IC, is small chip that can function as an amplifier, oscillator, timer, microprocessor, or even computer memory. An IC is a small wafer, usually made of silicon, that can hold anywhere from hundreds to millions of transistors, resistors, and capacitors. The IC used in this project is the LM3914 [3].

\section{Antenna}

Two types of antennas are used in this project. They are external and internal antennas.

\section{External directional antenna}

This antenna provides significant signal strength gain. The antenna is oriented and located outside to provide the best possible signal, usually aligned with the nearest cell tower. The larger the external antenna the better the signal. A correctly oriented external antenna can provide better signal than the internal antenna on any mobile phone. A pictorial view of an external antenna is shown in the figure 2 below. 


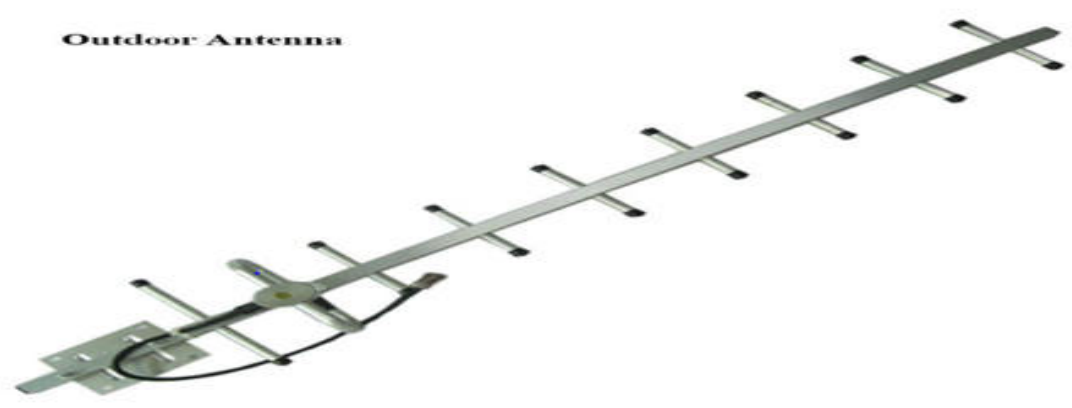

Figure 2. External antenna for the GSM signal booster

\section{Internal rebroadcast antenna}

Figure 3 below shows an internal monopole antenna, which can be used for rebroadcasting the signal internally. One major advantage of using a monopole antenna is that the signal will be equally distributed in all directions. This is subject to attenuation from obstacles. Since all radio antennas are intrinsically polarized, cell phones perform best when their antennas are oriented parallel to the booster's antenna - although within reasonable proximity to the booster's signal.

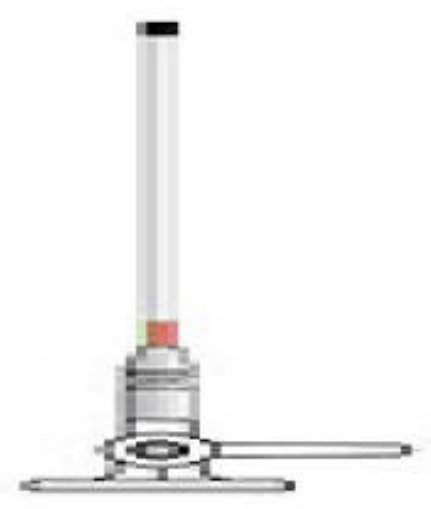

Figure 3. A monopole internal antenna.

Source: www.mobilenetworkguide.com.au

\subsection{Methodology}

It is necessary at this juncture to thoroughly examine the necessary steps required in the design process. As good practice in any system design, it is often best to follow methodical steps in designing all system components individually, and when it has been ascertained that the various components work as envisaged, the entire components is integrated as the final product. This is the Approach employed during the design phase of this signal booster. 
This is a hardware project and the methodology employed is practically inclined. It involves sourcing the components separately in the electronic market and building/cloning the device using the circuit diagrams provided in figures 4 and 5.

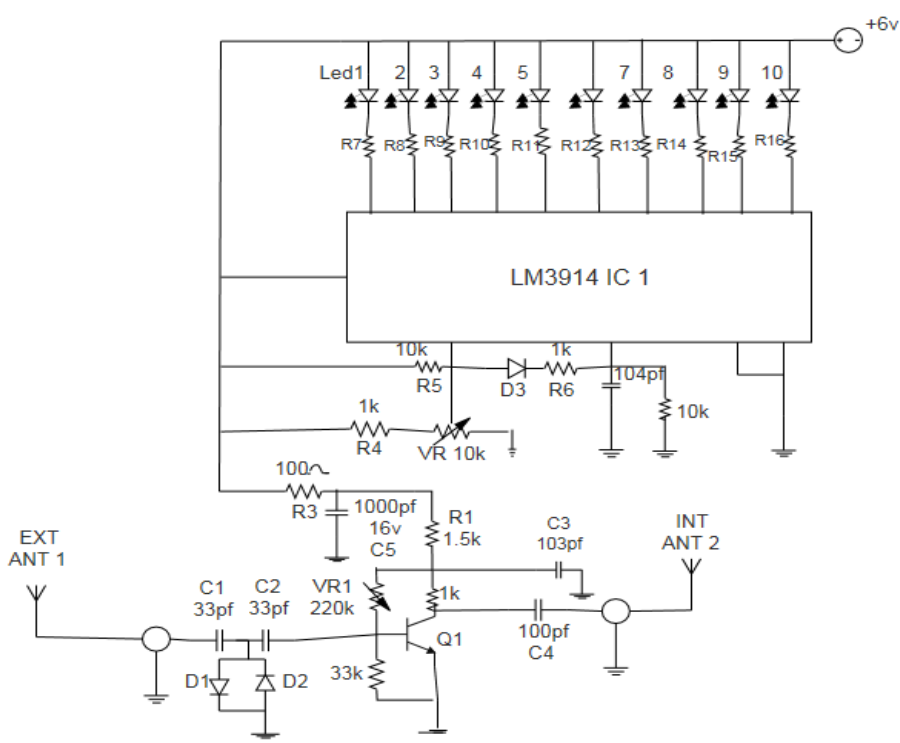

Figure 4. Complete circuit diagram of the signal booster

\section{Circuit Operation Principle}

The circuit shown in figure 4 is a complete circuit diagram of the GSM signal booster used to amplify the GSM signal. Q1 NPN RF transistor is connected in the circuit.

\section{Analysis}

\subsection{System Analysis}

In designing and constructing the GSM signal booster, it is necessary to understand the specification and operation of various components as well as how to use them to achieve the desired objectives. The components are grouped in stages which will be discussed accordingly.

\section{System Specification}

The design of GSM signal booster is based on the following technical specifications:

Input voltage: $6 \mathrm{Vdc}$

Input maximum current: 1-4 Ah

Input frequency: $1.7-1.8 \mathrm{GHz}$

Output frequency $1.7-1.8 \mathrm{GHz}$

The design analysis was conducted stage by stage and is mathematically explained as follows:

\section{Determination of Oscillator Stage Component}

In this stage, the RF transistor C555IA is used to amplify the incoming GSM signal

C555IA RF transistor 3V: 300mA

$\mathrm{FC}=3.5 \mathrm{GHz}$ NPN single PCP

Polarity is NPN

Type: VCE (sat) $\max (\mathrm{V}): 0.3$ 
Ic continuous (A): 0.3

$\mathrm{V}(\mathrm{BR}) \mathrm{CEO} \min (\mathrm{V}): 30$

hFE min: 90

hFE max: 270

Ft min (MHz): 3500

PTM max (W): 1.3

\section{Power Supply Unit}

From the given mathematical relations,

Input Power $=\mathrm{VI} \cos \theta=4 \times 2 \times 0.8=6.4 \mathrm{~W}$

\section{Voltage Indicator Unit}

The IC provides a decimal output by lighting the necessary LED based on the values of input voltage. The maximum measuring input voltage varies depending on reference voltage and supply voltage. The LM 3914 is a 10 stage measuring unit. This is shown in the internal circuit in figure 5 below. LM 3914 is basically a combination of 10 comparators. Each comparator is an OP amp with gaining reference voltage or the chip supply voltage.

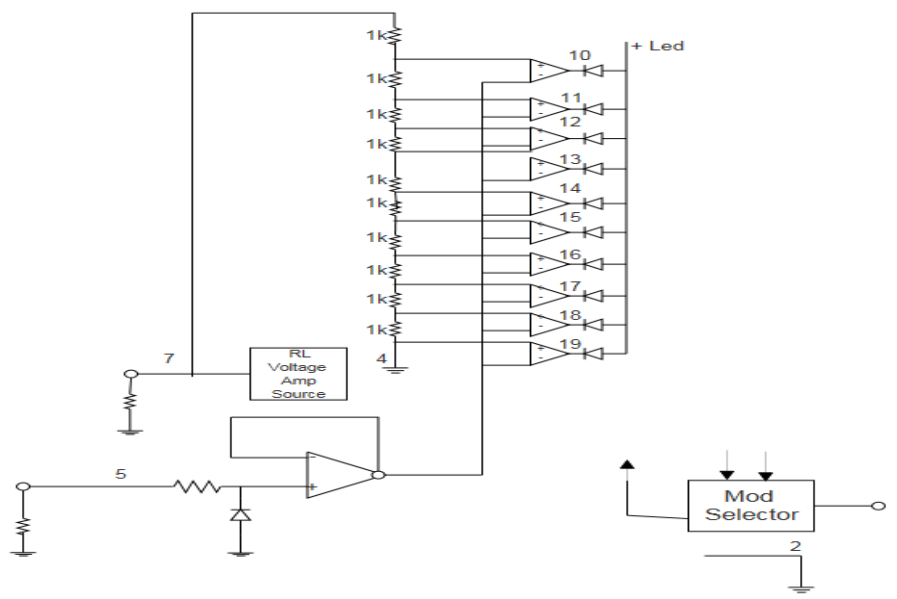

Figure 5. Internal circuit diagram of LM 3914

This must always be kept in mind for achieving a higher measuring voltage keeping the input constant we are going to use resistance voltage divider circuit. Consider the circuit formed by the network shown in figure 6 below:

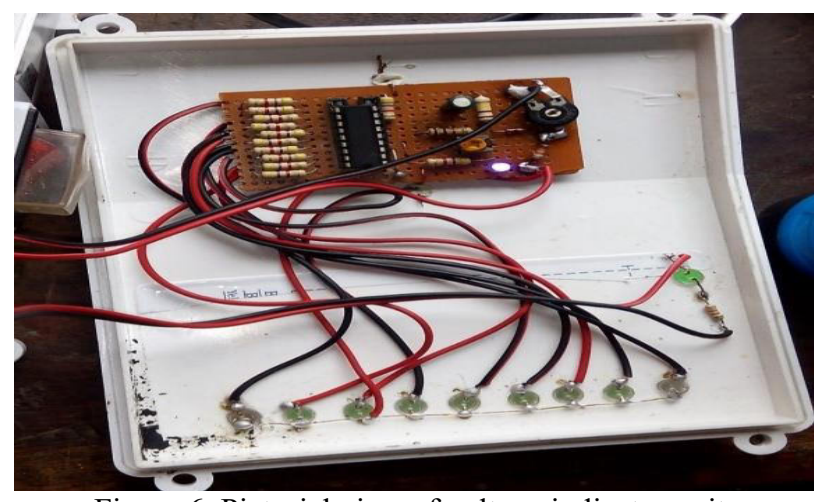

Figure 6. Pictorial view of voltage indicator unit. 


\section{Discussions and Results}

\subsection{System Implementation}

This section deals with the construction and testing of the GSM signal booster. At this stage of the project, all hardware components are brought together and cloned using the circuit diagram as a guide. A breadboard and wire wrapped connections were used initially. This was done so that it would be easier to change connections later. Great care was taken here bearing in mind that any wrong placement of components could lead to the malfunctioning of the device. The project was assembled temporarily on bread board to test how the circuit will operate with the design layout. The circuit was found to work according to the design specifications.

It is best to start up the construction by drawing up a neat wiring plan which shows the layout of the components on veroboard and how they were made with standard wires to fit neatly into the matrix in the veroboard. All wire tips and components leads were well cleaned and tinned. The surface of the vero board was slightly sandpapered to remove dirt and unwanted particles from the surface. All the copper tracks either full or of any lengths which were not used were broken off by scrapping thereby separating them from making contacts with the continuity parts of the circuit.

Where a component is needed to connect to another, a copper wire of very negligible resistance (jumper) is used. After the circuit was fully constructed and observations are made, the circuit is now ready for testing.

\section{Tools Used During the Construction}

A list of tools used in the project work are as follows:

i. Soldering iron $(60 \mathrm{~W})$

ii. Wire stripper for small conductors

iii. Sucker

iv. Long nose pliers

v. Soldering led

vi. Side cutter

vii. Pliers

viii. Digital multimeter etc.

The internal arrangement of components within the casing is shown pictorially in figure 7 below.

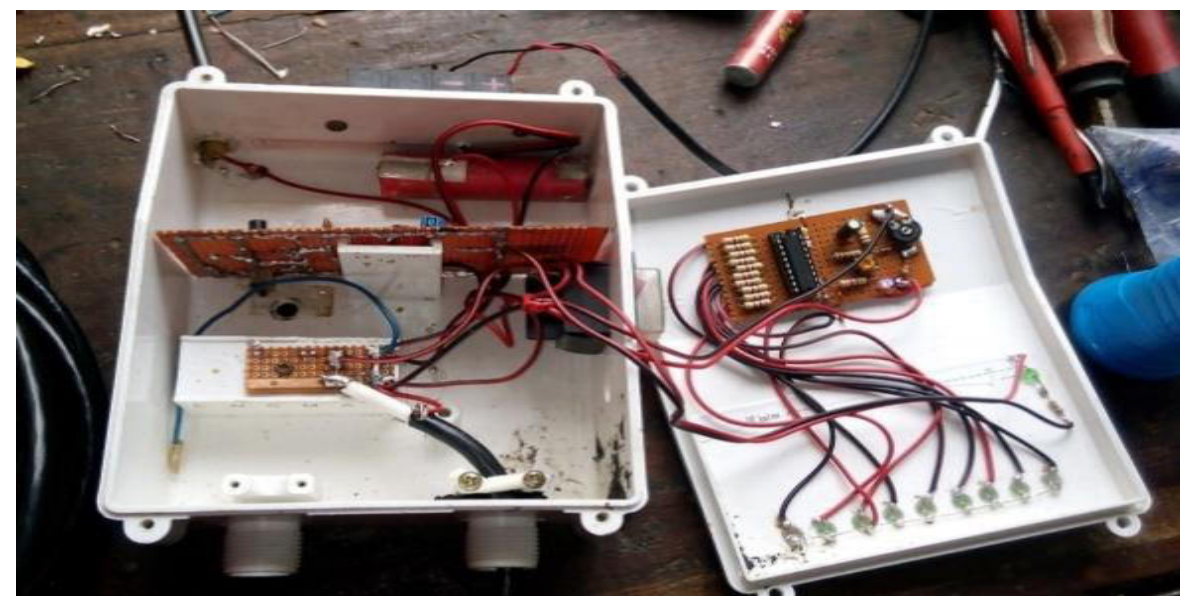

Figure 7. Pictorial view of the internal components of the GSM signal booster 


\section{Casing}

The booster is made up of a plastic casing. All the boards are fastened to the casing using screws. This is shown in figure 8 below.

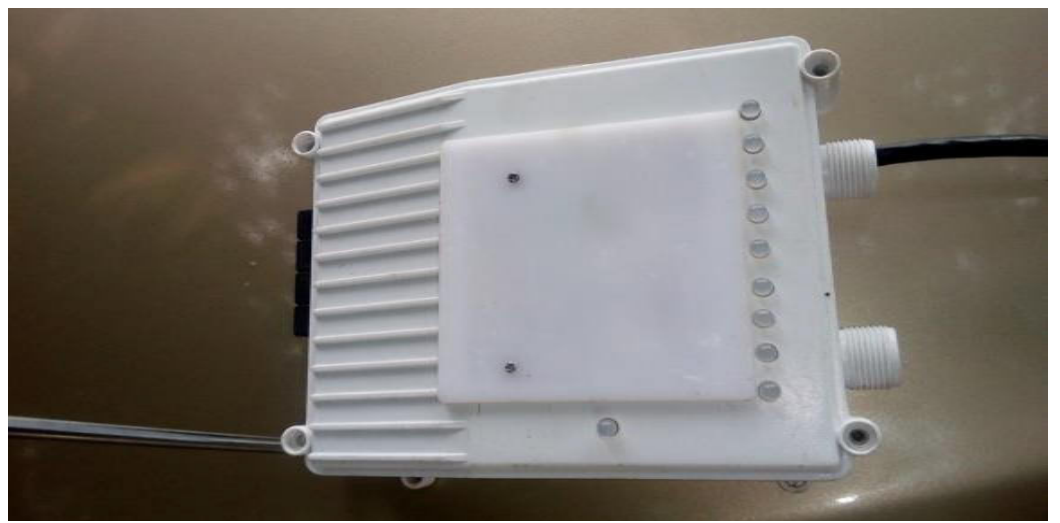

Figure 8. the casing of the signal booster

\section{Installing the Signal Booster}

The cellular booster should be installed in a location that is properly ventilated and not exposed to excessive heat, moisture and/or direct sunlight. An excellent area would be on a wall. It should be mounted in an easily accessible area so it is easy to perform general maintenance with the coaxial cable connections, dip switch settings and power adaptor. All cables and antennas should be securely connected before commissioning the system.

\section{Powering Up the Cellular Booster}

Once all the necessary precautions were taken, the cellular booster was then powered on using the ON/OFF switch provided on the right side of the booster.

\subsection{Measurement and Testing}

After the circuit was fully constructed and observations are made, the circuit is now ready for testing.

\section{Measuring Signal Strength}

Measuring the signal strength of a particular GSM service provider is not as simple as just viewing the number of service bars produced by a mobile phone network signal. There are two ways of measuring mobile signal on a cell phone. They include:

i. Using the signal bars on your mobile device:

The signal bars on your mobile device will offer a very basic interpretation of the mobile signal and also the signal quality. Each phone manufacturer calculates how many bars to display differently (see figure 9 below). The signal bars do not always mean you can make a call. It is possible to have signal bars and not be able to place a call due to network congestion.

The bars on your phone are a representation of both signal strength and the quality of the signal. iPhone-iOS 7 takes into account the signal strength, signal quality and also the number of people using the base station to determine how many bars are displayed [4]. 


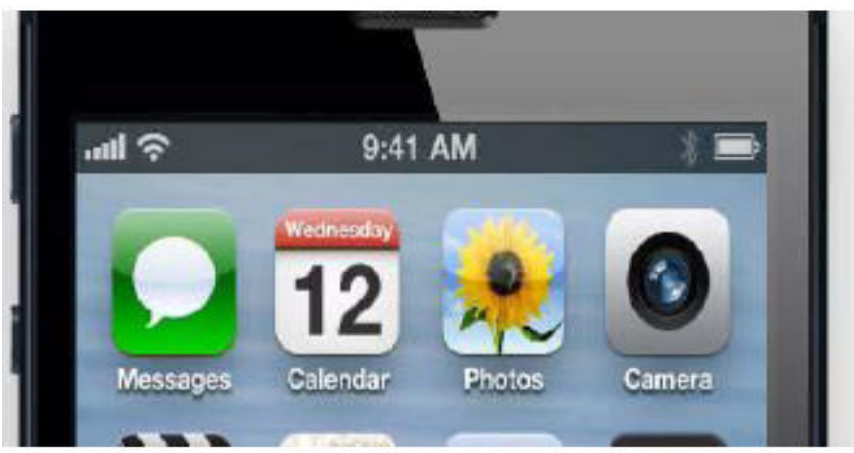

Figure 9. Signal bars on your mobile device www.mobilenetworkguide.com.au

This helps to prevent a user thinking they can place a call when they have several bars.

ii. Obtaining exact readings:

The other method of measuring signal strength is byusing exact readings from mobile phones. In this work, we downloaded a signal App called Network Signal Info from Play Store (see figure 10 below).

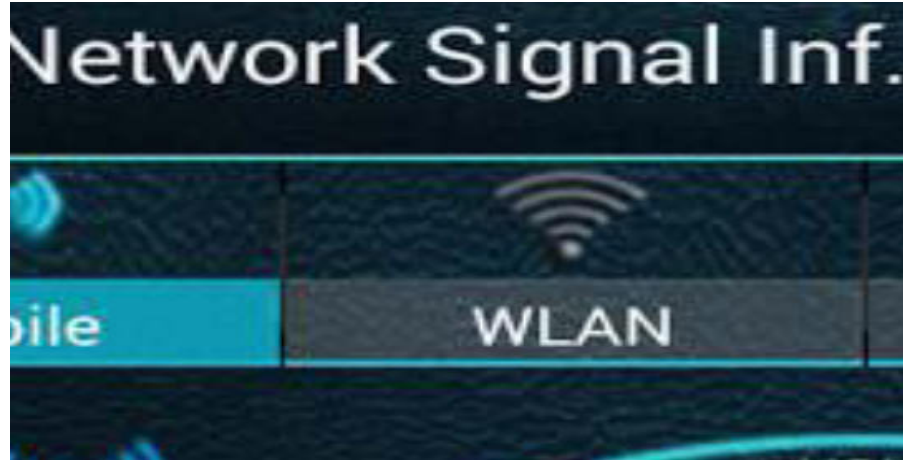

Figure 10. Signal App, a Network Signal Info from play Store

\section{Signal Strength - GSM \& 3G/HSPA (RSSI)}

Applicable to GSM and 3G/High Speed Packet Access (HSPA) networks. The exact signal strength, often called Received Signal Strength Indication (RSSI), is measured in $\mathrm{dBm}$ (sometimes referred to as dBmw or decibel miliwatts).

The $\mathrm{dBm}$ scale is roughly between -50 and $-120 \mathrm{dBm}$, with -50 being perfect signal and -120 being when you fall off the network. RSSI measures both the usable signal and the noise in a single figure [4].

-50 to $-75 \mathrm{dBm}-$ High Signal

-76 to $-90 \mathrm{dBm}$ - Medium Signal

-91 to $-100 \mathrm{dBm}$ - Low Signal

$-101 \mathrm{db}$ to $-120 \mathrm{dBm}-$ Poor Signal

\section{Signal Strength - 4G/LTE (RSRP)}

LTE signal strength is measured on a different scale from 3G/HSPA, it is measured in Reference Signal Received Power (RSRP). This often ends up being around -20dBmlower than RSSI, so -100dBm (RSSI) would equate to around $-120 \mathrm{dbm}(\mathrm{RSRP})$. 
RSRP does a much more accurate job of measuring signal strength than RSSI, as it excludes noise and interference on the network, measuring just the usable portion of the signal. Just because RSRP signals appear lower, itwill not mean the signal is worse.

$-75 \mathrm{dBm}$ and $-88 \mathrm{dBm}$ is a strong signal

$-89 \mathrm{dBm}$ and $-96 \mathrm{dBm}$ is a very good signal

$-97 \mathrm{dBm}$ and $-105 \mathrm{dBm}$ is good

$-106 \mathrm{dBm}$ and $-112 \mathrm{dBm}$ is fair

$-113 \mathrm{dBm}$ and $-125 \mathrm{dBm}$ is poor

\section{Signal Quality}

Signal to Noise Ratio (SNR): This is a measurement that compares the signal strength to the level of background noise. The higher the SNR the better the signal quality will be. The SNR reading will be automatically calculated by the base station in $\mathrm{dB}$. On the SNR scale 4 is poor and 25 is great.

Arbitrary Strength Unit (ASU): This is a value that is proportional to RSRP. The higher the number, the better your signal quality. ASU $=$ RSRP +140 , so if you have a RSRP of $-100 \mathrm{dBm}$, then the ASU will be 40 .

Ec/Io: This is very similar to SNR above, this measurement is the ratio of signal to interference. With the best being around -10 and the worst quality being around -40 .

Reference Signal Received Quality (RSRQ): This is the ratio of usable signal to noise and interference measured in $\mathrm{dBm}$. RSRQ $=$ RSRP - RSSI

Gain: the gain of an antenna is its effectiveness in focusing radio frequency energy in a particular direction. The higher the gain the more focused the antenna is and the narrower the field of reception. Antenna gain is measured in $\mathrm{dBi}$ or $\mathrm{dBd}$ and the greater the gain the better the signal should be [4].

\section{Conclusion}

This paper aimed at designing and constructing GSM signal booster had the singular purpose of enhancing the signal level of most cells phones in a particular vicinity so as to make it easier for the mobile phones to make and receive calls without much hitch. The signal booster system was able to trap wireless signals through the antenna and amplify it resulting in an overall gain.

This is to make GSM network available to everyone irrespective of height, terrain and location and in the long term facilitates business activities in small, medium as well as large scale enterprises in villages and cities and to take the radio link available in Base stations to areas where they are highly needed in order to make calls and use data in all terrains where mobile phones are used.

With the GSM mobile booster device, users can now enjoy a seamless, uninterrupted and reliable data communication.

They can also obtain/enjoy stronger signal strengths across longer ranges. This booster design is new and made from local components which are affordable.

\section{Recommendations}

This work should serve as an aid to any subsequent work on the design and construction of a mobile phone signal booster. The work should not end here; companies, entrepreneurs and investors should take it up from here so that we can enjoy seamless, uninterrupted and reliable data communication across long ranges. With this work improved upon, a device that will be very useful can be created which will rival other available boosters in the market, especially with respect to its affordability.

\section{REFERENCES}

[1] Nicholas W. Scott, (2011) Study of Cellular Phone Detection Techniques (Pp 1,2,5)

[2] "Wi-Fi Alliance: Organization". http://www.wifi. org/organization.php. 
[3] B. L.Theraja (2010) Electrical Technology, 5th edition.

[4] www.mobilenetworkguide.com.au (Pp 9-14).

[5] A. P. Omoniyi (2011), GSM Telecommunications Design and Methodology (Unpublished) (Pp 2-3, 13-30).

[6] www.tech-faq.com>cell-phone-signal-booster.html.

[7] Andrew M. Seybold (July 2010), A white paper Prepared for Wilson Electronics seybold@andrewseybold.com.

[8] https://www.statista.com/statistics/274774/forcast-of-mobilephone-users-worldwide/

[9] www.wilsonelectronics.com.

[10] https://www.livescience.com/46021-what-is-a-transistor.html.

[11] Prof. Neal Patwari (2011), Wireless Communication Systems Lecture Notes, University of Utah.

[12] N. Abramson. The aloha system-another alternative for computer communications. In Proceedings of the Fall Joint Computer Conference, pages 281-285, 1970.

[13] https:/www.slideshare.net/mobile/raafatyounis/5432-cellularnetwork 